\title{
Fiber-Coupled Surface Plasmon Polariton Excitation in Imprinted Dielectric-Loaded Waveguides
}

\author{
Andreas Seidel, ${ }^{1}$ Jacek Gosciniak, ${ }^{2}$ Maria U. Gonzalez, ${ }^{3}$ Jan Renger, ${ }^{3}$ Carsten Reinhardt, ${ }^{1}$ \\ Roman Kiyan, ${ }^{1}$ Romain Quidant, ${ }^{3}$ Sergey I. Bozhevolnyi, ${ }^{2}$ and Boris N. Chichkov ${ }^{1}$ \\ ${ }^{1}$ Laser Zentrum Hannover e.V., Hollerithallee 8, 30419 Hannover, Germany \\ ${ }^{2}$ University of Southern Denmark, Niels Bohrs Alle 1, 5230 Odense M, Denmark \\ ${ }^{3}$ Institut de Ciencies Fotoniques (ICFO), Mediterranean Technology Park, Castelldefels, 08860 Barcelona, Spain
}

Correspondence should be addressed to Andreas Seidel, a.seidel@lzh.de

Received 21 April 2010; Accepted 17 June 2010

Academic Editor: Qihuang Huang Gong

Copyright (c) 2010 Andreas Seidel et al. This is an open access article distributed under the Creative Commons Attribution License, which permits unrestricted use, distribution, and reproduction in any medium, provided the original work is properly cited.

\begin{abstract}
We present fiber-coupled dielectric-loaded plasmonic waveguides for $1.55 \mu \mathrm{m}$ telecom wavelength fabricated by two-photon polymerization and nanoimprint lithography. The waveguide structures include $100-\mu \mathrm{m}$-long plasmonic waveguides connected on both ends to tapered dielectric waveguides used for end-fire coupling with optical fibers. The excitation of plasmonic waveguides is verified via polarization-resolved measurements of the overall transmission, demonstrating thereby that this technology is suitable in principle for the integration of plasmonic components into fiberoptics. Loss mechanisms are investigated and improvements suggested to reduce transmission losses and consequently increase the viability of practical application.
\end{abstract}

\section{Introduction}

Photonic components utilizing surface plasmon polariton (SPP) modes supported by various metal-dielectric configurations have been attracting a great deal of attention, because of promising applications in sensing and on-chip data transmission [1-3] and the advantage of utilizing the same metallic circuitry for both guiding the radiation and controlling its guidance [4]. An important aspect, however, is compatibility between different configurations. Most optical systems and optical data transmission networks are fiberbased, so it is particularly important to provide the ability to interconnect plasmonic waveguides and optical fibers for integration purposes.

In this paper, we present plasmonic-optical hybrid waveguides fabricated by nanoimprint lithography from master structures made by two-photon polymerization technique. The plasmonic waveguides are interconnected with tapered dielectric waveguides suitable for end-fire coupling with optical fibers. The layout of the structures is derived from a previous design that has been studied in some detail $[5,6]$, but was not suited to a fiber-based environment due to the impossibility of approaching the structures with a fiber close enough for coupling. This has been remedied by the inclusion of optical waveguides in the design that can be coupled to optical fibers (Figure 1(a)).

The substrate used is a quartz glass slab measuring $24 \times 4 \mathrm{~mm}$. The refractive index of the glass is 1.44 at a wavelength of $1.55 \mu \mathrm{m}$. In the center of the glass slab, there is a longitudinal gold stripe measuring $125 \mu \mathrm{m}$ across and several millimeters long, which is patterned using electron beam lithography and a lift-off process. The gold layer deposited by evaporation is $50 \mathrm{~nm}$ thick. For the waveguide material we use the nanoimprint lithography resist mr-NIL 6000 made by the company micro resist technology $\mathrm{GmbH}$ in Berlin. The refractive index of the polymer waveguide is 1.523 according to the manufacturer. The waveguides run across the sample at a right angle to the gold stripe and are thus also $4 \mathrm{~mm}$ long. The waveguides consist of optical waveguide parts for in- and outcoupling and a dielectricloaded surface plasmon-polariton waveguides (DLSPPW) [5, 7-9] section $100 \mu \mathrm{m}$ long in the center, where the gold layer is located. It is necessary to have single mode waveguiding from fiber to fiber, and this places restraints on the possible 

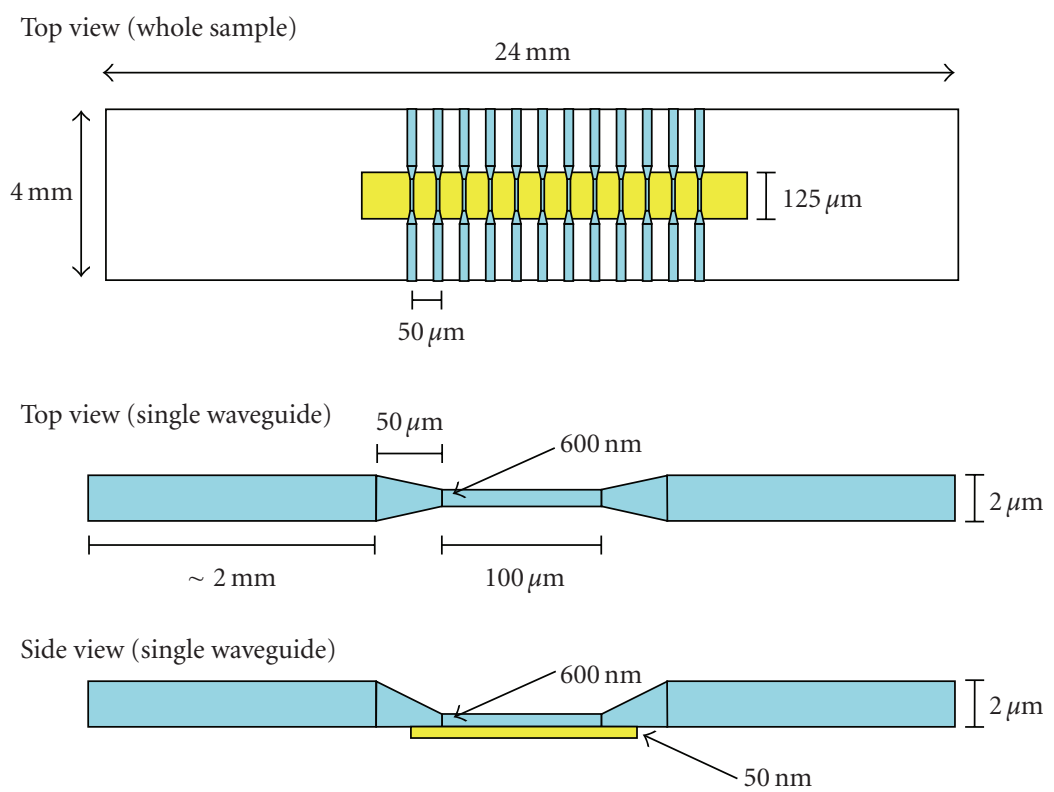

(a)

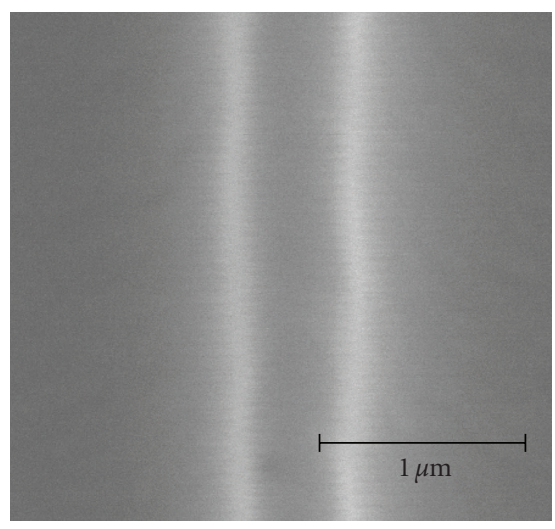

(b)

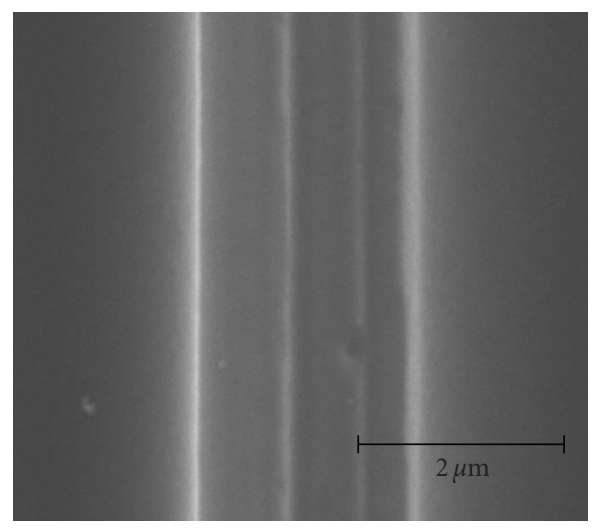

(c)

FIGURE 1: (a) A schema showing the layout of a sample with plasmonic waveguides. The waveguides consist of optical waveguides at either end which taper down to a 100 micrometer long plasmonic waveguide only $600 \mathrm{~nm}$ wide. (b) SEM image of the DLSPPW section. (c) SEM image of the optical waveguide section.

dimensions of our waveguides. The DLSPPW section has a rectangular $0.6 \times 0.6 \mu \mathrm{m}$ cross-section, which is singlemode for $1.55 \mu \mathrm{m}$ light $[5,6]$. The optical section of the waveguide has a rectangular $2 \times 2 \mu \mathrm{m}$ cross-section, which also makes it single-mode, as was obtained by numerical simulations [10]. To have a single-mode optical waveguide is important, since a multimode waveguide would incur very large losses when coupling to the single-mode plasmonic waveguide. The taper between the two sections is $50 \mu \mathrm{m}$ long.

Sample fabrication is a multistep process. In the first step, the waveguide structures are fabricated by two-photonpolymerization (2PP) on a highly-polished glass mirror substrate using custom-made ormosil [11] as a photo resist. $2 \mathrm{PP}$ is a very versatile micro- and nanopatterning technique $[12,13]$ and is the only suitable technique for the fabrication of these structures due to their geometry and dimension. Traditional UV mask lithography, which would provide the necessary resolution of $600 \mathrm{~nm}$, cannot handle the threedimensional aspect of the required structures and stereo lithography, while being able to make 3D structures,it lacks resolution.

After 2PP fabrication of the masters, a stamp is made for nanoimprint lithography. The stamp material used is polydimethylsiloxane (PDMS). The stamp is used in combination with an imprint resist to create a copy of the master structures on the substrates using mr-NIL 6000 as resist material. This procedure for making functional plasmonic components by this method has already been shown to work well [14]. After stamping, overhanging edges of imprint resist are removed using a micromechanical cleavage technique. 


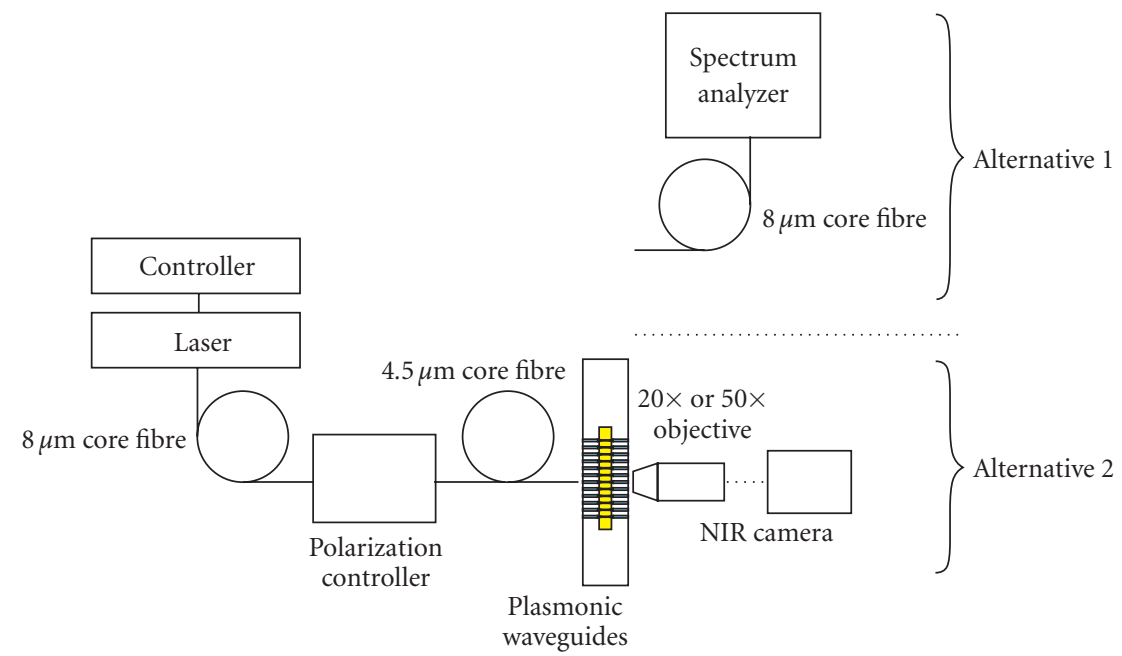

FIGURE 2: A schema showing the layout of the experimental setup.

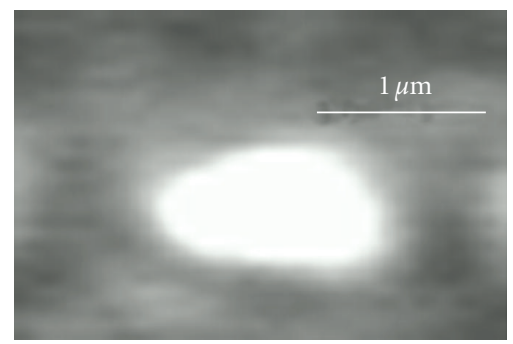

(a)

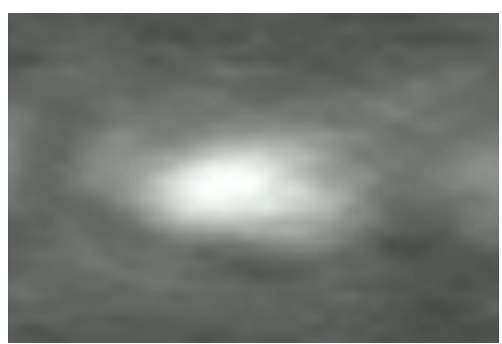

(b)

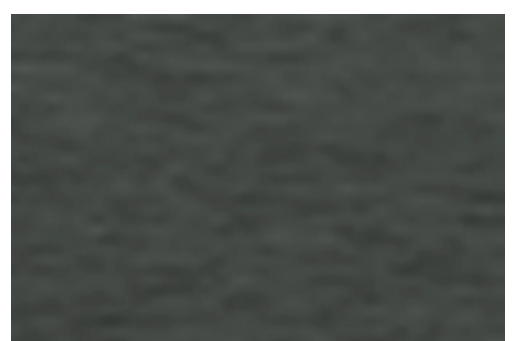

(c)

FIGURE 3: Images from the near infrared camera show the output of the DLSPPW waveguides at different polarizations of input light. (a) $0^{\circ}$ Polarization (b) $45^{\circ}$ Polarization (c) $90^{\circ}$ Polarization. As can be seen, output intensity decreases drastically with polarization.

\section{Experimental Results}

After fabrication, the samples are characterized by exciting plasmons in an end-fire configuration. A schema of the experimental setup is shown in Figure 2. We use a fibercoupled $1550 \mathrm{~nm}$ laser. It is connected to a polarization controller by a standard $8 \mu \mathrm{m}$ core fiber. Following this, there is a small-core fiber to match the mode volume to the optical waveguides on the sample. Using a $4.5 \mu \mathrm{m}$ mode-field diameter fiber provides much better mode matching than standard fibers when we take into account that the on-sample optical waveguides are only $2 \mu \mathrm{m}$ wide. The end of the fiber is brought within several microns of the waveguide entrance. Coupling can be observed from above by a microscope, which is not shown in the schema.

We present results of both qualitative and quantitative measurements. Two alternatives exist for analyzing and viewing the outcoupled light in our setup. The first possibility is to use a microscope objective and near infrared camera to image the far end of the sample. This is convenient for adjusting the incoupling efficiency and monitoring the experiment and is used for qualitative measurements. The second possibility is to couple the light back into a fiber to a spectrum analyzer. This is used for quantitative measurements.

Figure 3 shows camera images gained from the first method. The camera uses an automatic gain adjustment so the images are not comparable in a quantitative way. Transmitted light through the waveguide is shown. Since the DLSPPW section of the waveguide is too small to allow an optical mode, light must be converted into a plasmon and then converted back to be visible. A strong polarization dependency is expected. At this point, we will set the reference point for polarization with $0^{\circ}$ coincidental with TM polarization that is, the electrical field is perpendicular to the sample surface and is suitable for exciting SPP modes. The results show this. Figure 3(a) shows the transmission at $0^{\circ}$ polarization. Here, maximum intensity is expected and observed. Figure $3(\mathrm{~b})$ shows the waveguide at $45^{\circ}$ polarization and $3(\mathrm{c})$ finally at $90^{\circ}$. As can clearly be seen, the intensity of transmitted light drops drastically as polarization is turned and the plasmonic mode cannot be excited. The light visible in the images not originating from the waveguide facet is due to light scattered at the coupling point of plasmonic to optical waveguide, which is scattered by irregularities on the sample edge. 


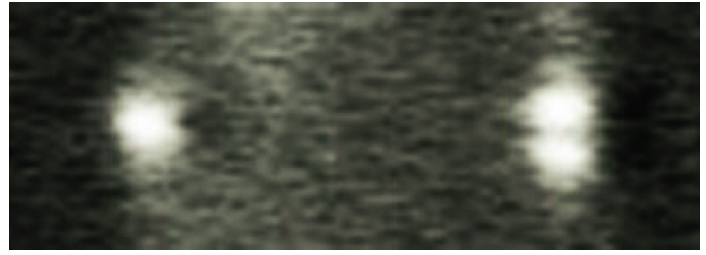

(a)

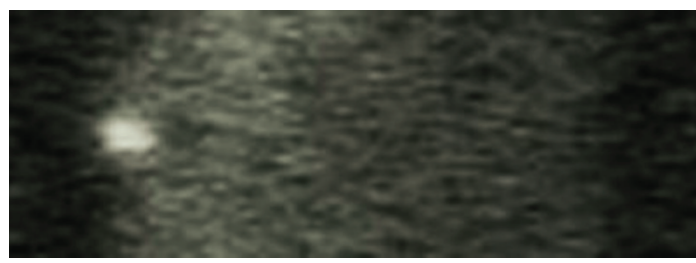

(c)

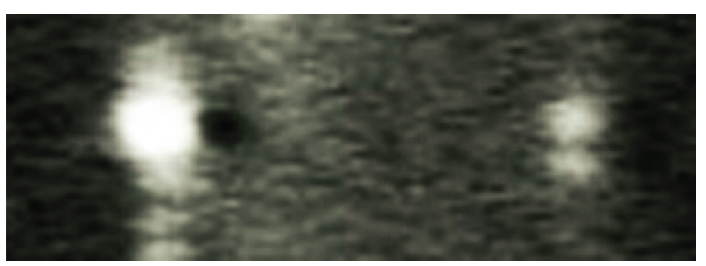

(b)

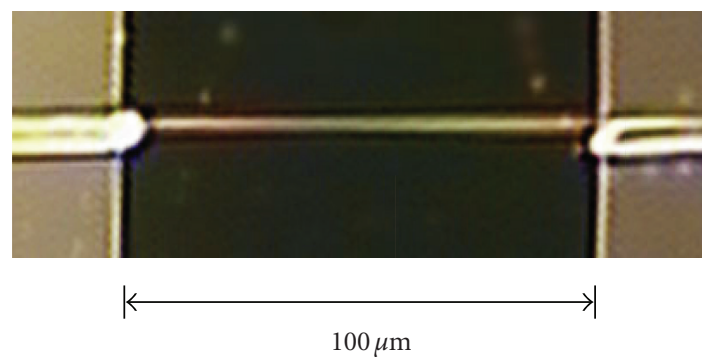

(d)

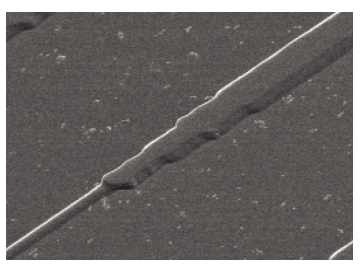

(e)

FIGURE 4: Images from the near infrared camera showing a top-down view of the sample centered on the DLSPPW part of the waveguide. Left side is the incoupling, right side is the outcoupling. (a) $0^{\circ}$ Polarization (b) $45^{\circ}$ Polarization (c) $90^{\circ}$ Polarization. While the incoupling spot stays bright, the outcoupling spot rapidly decreases in intensity, showing that the plasmonic mode is suppressed with changing polarization. (d) Optical microscope image of waveguide showing gold stripe and DLSPPW section shown for comparison. (e) SEM image (same scale as optical images) showing the transition from plasmonic to optical waveguide at an angle of $45^{\circ}$.

Further observations confirm this. Figure 4 shows the samples from a top-down view using the near infra-red camera, focused on the DLSPPW section of the waveguide, that is, the plasmonic part. The gold stripe is visible in the images as a bright band. Light is incoupled from the left in the images. The two bright spots originate from scattering at the tapers where the waveguide changes from purely optical to plasmonic and back. Polarization is changed from $0^{\circ}$ Figure $4(\mathrm{a})$ through $45^{\circ}$ Figure $4(\mathrm{~b})$ to $90^{\circ}$ Figure 4(c). Figure 4(d) shows an optical microscope image of the structure for comparison and Figure 4(e) shows the transition region from optical to plasmonic waveguide in detail. We observe that the incoupling spot remains bright at all polarizations, while the outcoupling spot rapidly decreases in intensity. This is due to the plasmonic mode not being excited at $90^{\circ}$ polarization, and transmission thus suppressed. As before, the camera uses automatic gain adjust, which accounts for inconsistencies in overall brightness.

The spectrum of the transmitted mode was measured and is shown in Figure 5(a). This provides an easy means to quantify the intensity of the output mode. Quantitative measurements of the transmission changes with polarization were performed. Results are shown in Figure 5(b). As can be seen, the graph follows a cosine pattern, which is in line with expectations.
Measurements show average losses of $49 \pm 2 \mathrm{~dB}$. Propagation losses in the DLSPPW section, which is 100 micrometers long, can be calculated and amount to $8 \mathrm{~dB}$, a figure which is backed up by early experiments [14]. The remainder of the losses can be attributed to several causes. One cause is the loss at the insertion point between the optical fiber and the optical waveguide due to scattering from the waveguide end face. The waveguide end faces are not perfectly flat or perfectly perpendicular to the waveguide and this causes a large amount of scattering. A further source of loss lies in the optical waveguides themselves due to scattering and waveguide imperfections. And finally there are losses at the coupling between optical and DLSPPW mode.

To quantify the coupling efficiency from optical to plasmonic mode and back, reference measurements were undertaken with all-optical waveguides under the same conditions. The optical waveguides use the same substrate as before but without gold. The waveguides are $4 \mathrm{~mm}$ long and have a $2 \times 2 \mu \mathrm{m}$ rectangular cross-section, identical to the optical in- and outcoupling waveguides on the plasmonic samples. Measurements showed that the insertion and optical guiding losses amount to $37.5 \mathrm{~dB} \pm 0.7 \mathrm{~dB}$ on average. This is a relatively high loss for an optical waveguide, which can be explained by several things. One source of losses is the surface roughness along the length of the waveguide. 


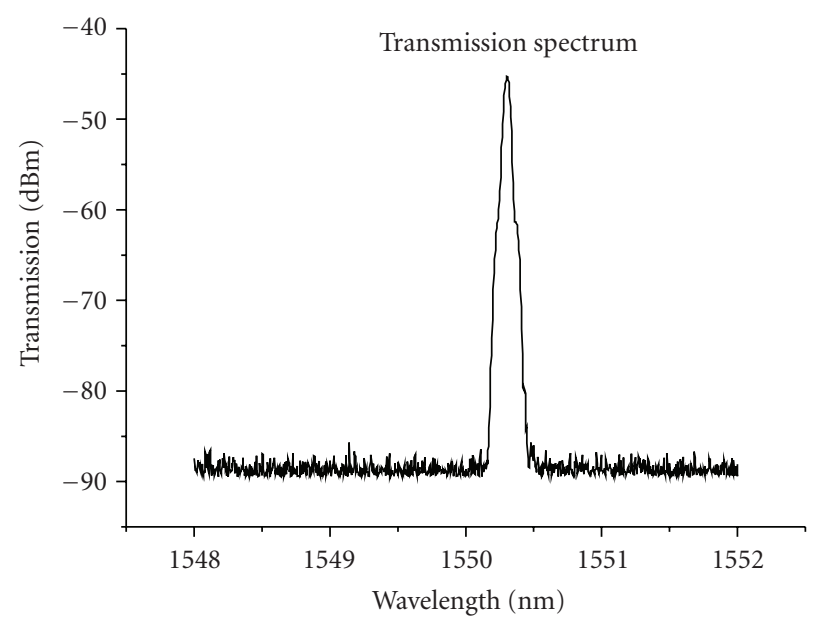

(a)

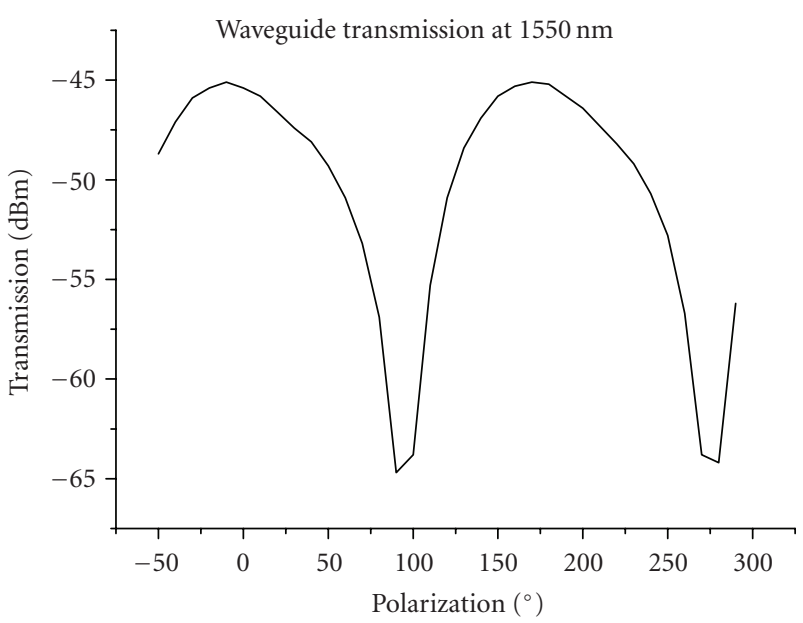

(b)

Figure 5: (a) The spectrum of the transmitted mode. (b) Graph showing the dependency of transmission through the waveguide on polarization - a characteristic of the DLSPPW mode.

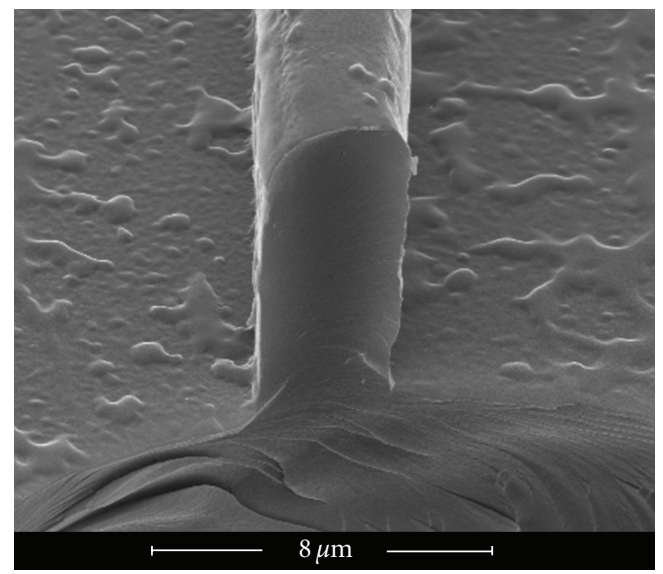

FIGURE 6: SEM image showing the cleaved end of an optical waveguide. As can be seen, the cut is quite clean, however, it is not perfectly perpendicular to the waveuide nor is it perfectly flat, and in addition there are some minor scratches in the waveguide. All of these features have an impact on the outcoupling of light from the waveguide and all of them can differ from waveguide to waveguide.

The surface of the waveguide is not perfectly smooth due to fabrication irregularities, as can be seen in the images in Figures 1(c) and 4(d). Another source of losses is the low reproducibility in quality of the cleaved ends of the waveguides, already mentioned briefly above. An image of a typical cleaved waveguide end can be seen in Figure 6. The face of the waveguide ends should be as plane as possible, and cut as close as possible to a $90^{\circ}$ angle. Unfortunately with current technology, as can be seen in figure 6 , results are often produced that do not perfectly match the ideal conditions. Typical problems are that the cleavage is not perfectly perpendicular to the waveguide and that minor scratches remain in the waveguide face. These problems could be solved by polishing the waveguide ends, however, this is difficult considering that the relatively soft polymer waveguide is located on top of a relatively hard substrate and polishing methods that are currently available would be overabrasive on the polymer, leading to an unsatisfactory result. Research is ongoing, however, to make polishing the waveguide ends an option in the future. An additional source of loss is the coupling from optical fiber to waveguide and back again. Currently, this is achieved only by careful alignment through a very narrow air gap, and clearly an amount of light is lost in this way. Experiments could be made in the future with index-matching oil to provide a link between optical fiber and waveguide.

If we take this data together, the calculated $8 \mathrm{~dB}$ loss for the DLSPPW mode and the $37.5 \pm 0.7 \mathrm{~dB}$ loss for the optical waveguides and combine this with the experimental results for the DLSPPW waveguides, we can estimate the coupling losses between optical waveguide and DLSPPW waveguide and back again to lie at $3.5 \pm 2 \mathrm{~dB}$.

\section{Conclusion}

We have shown excitation of DLSPPW modes in end-fire coupling configuration in fiber-coupled waveguides. The transmitted intensity was compared to reference measurements on all-optical waveguides and this resulted in a range for the coupling loss from optical to plasmonic mode in our structures. There are several points which could be improved to increase the overall efficiency of these waveguides. One part is certainly the coupling between optical fiber and optical waveguide. Here, future research will focus on finding ways to guarantee a flat waveguide end, possibly by polishing, and on ways to reduce scattering by using index-matching oil or similar materials to connect fiber and waveguide. Another area of focus for future research on this topic is the design of the optical waveguide to DLSPPW section tapers. Currently the shape and form of the tapers still leaves room for optimization and research in this area has already begun. 


\section{Acknowledgments}

The authors would like to acknowledge the financial support of the PLASMOCOM Project (EC FP6 IST 034754 STREP) and the Center for Quantum Engineering and Space-Time Research (QUEST) for funding this paper.

\section{References}

[1] J. Homola, S. S. Yee, and G. Gauglitz, "Surface plasmon resonance sensors: review," Sensors and Actuators, B, vol. 54, no. 1, pp. 3-15, 1999.

[2] T. W. Ebbesen, C. Genet, and S. I. Bozhevolnyi, "Surfaceplasmon circuitry," Physics Today, vol. 61, no. 5, pp. 44-50, 2008.

[3] S. I. Bozhevolnyi, Ed., Plasmonic Nanoguides and Circuits, Pan Stanford, Singapore, 2009.

[4] T. Nikolajsen, K. Leosson, and S. I. Bozhevolnyi, "Surface plasmon polariton based modulators and switches operating at telecom wavelengths," Applied Physics Letters, vol. 85, no. 24, pp. 5833-5835, 2004.

[5] T. Holmgaard and S. I. Bozhevolnyi, "Theoretical analysis of dielectric-loaded surface plasmon-polariton waveguides," Physical Review B, vol. 75, no. 24, Article ID 245405, 2007.

[6] T. Holmgaard, S. I. Bozhevolnyi, L. Markey, and A. Dereux, "Dielectric-loaded surface plasmon-polariton waveguides at telecommunication wavelengths: excitation and characterization," Applied Physics Letters, vol. 92, no. 1, Article ID 011124, 2008.

[7] C. Reinhardt, S. Passinger, B. N. Chichkov, C. Marquart, I. P. Radko, and S. I. Bozhevolnyi, "Laser-fabricated dielectric optical components for surface plasmon polaritons," Optics Letters, vol. 31, no. 9, pp. 1307-1309, 2006.

[8] A. V. Krasavin and A. V. Zayats, "Passive photonic elements based on dielectric-loaded surface plasmon polariton waveguides," Applied Physics Letters, vol. 90, no. 21, Article ID 211101, 2007.

[9] B. Steinberger, A. Hohenau, H. Ditlbacher et al., "Dielectric stripes on gold as surface plasmon waveguides," Applied Physics Letters, vol. 88, no. 9, Article ID 094104, 2006.

[10] K. Bierwirth, N. Schulz, and F. Arndt, "Finite-difference analysis of rectangular dielectric waveguide structures," IEEE Transactions on Microwave Theory and Techniques, vol. 34, no. 11, pp. 1104-1114, 1986.

[11] B. Bhuian, R. J. Winfield, S. OBrien, and G. M. Crean, "Investigation of the two-photon polymerisation of a Zr-based inorganicorganic hybrid material system," Applied Surface Science, vol. 252, pp. 4845-4849, 2006.

[12] J. Serbin, A. Ovsianikov, and B. Chichkov, "Fabrication of woodpile structures by two-photon polymerization and investigation of their optical properties," Optics Express, vol. 12, no. 21, pp. 5221-5228, 2004.

[13] R. Kiyan, G. Reinhardt, S. Passinger et al., "Rapid prototyping of optical components for surface plasmon polaritons," Optics Express, vol. 15, no. 7, pp. 4205-4215, 2007.

[14] A. Seidel, C. Ohrt, S. Passinger, C. Reinhardt, R. Kiyan, and B. N. Chichkov, "Nanoimprinting of dielectric loaded surfaceplasmon-polariton waveguides using masters fabricated by 2-photon polymerization technique," Journal of the Optical Society of America B, vol. 26, pp. 810-812, 2009. 

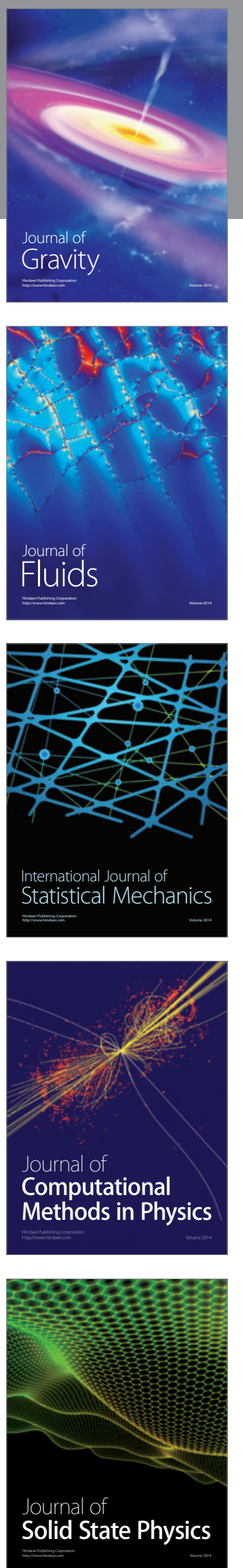

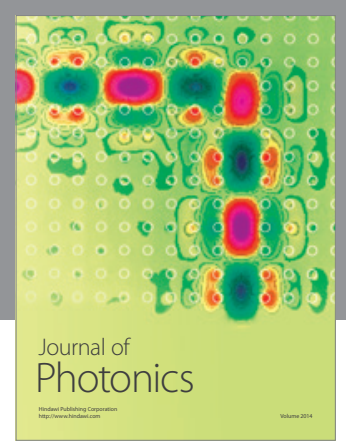

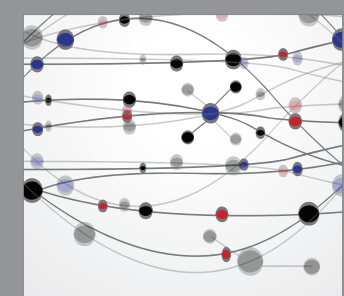

The Scientific World Journal
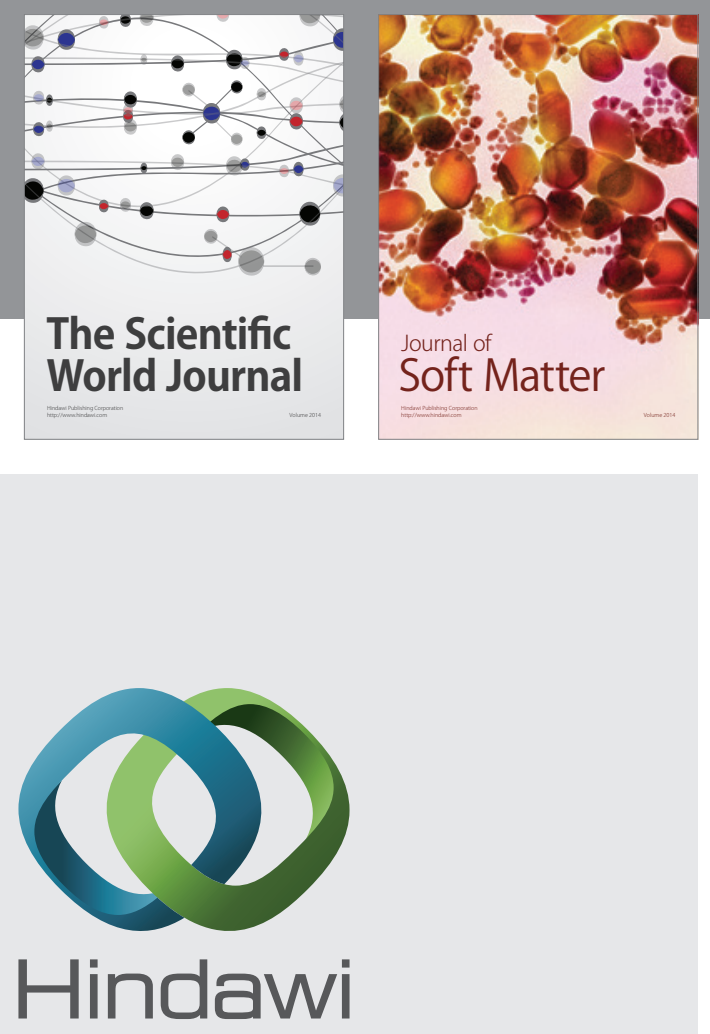

Submit your manuscripts at

http://www.hindawi.com
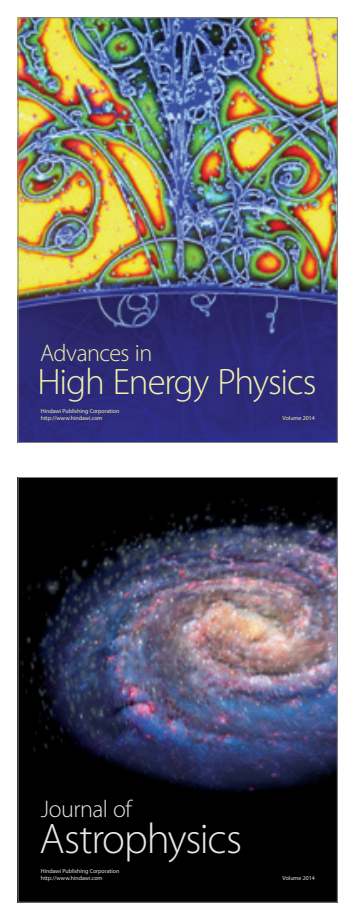
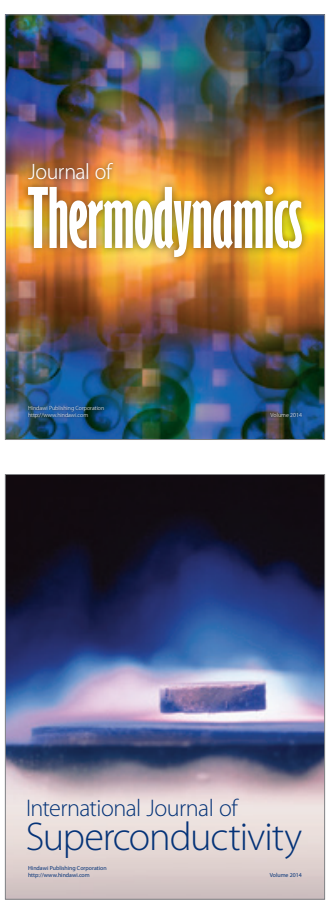
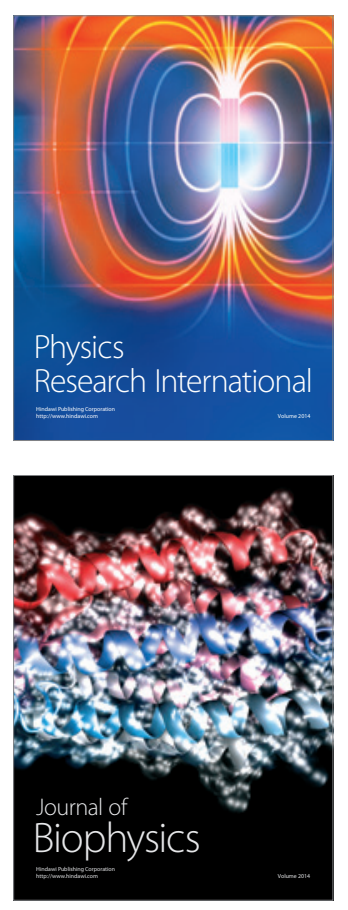
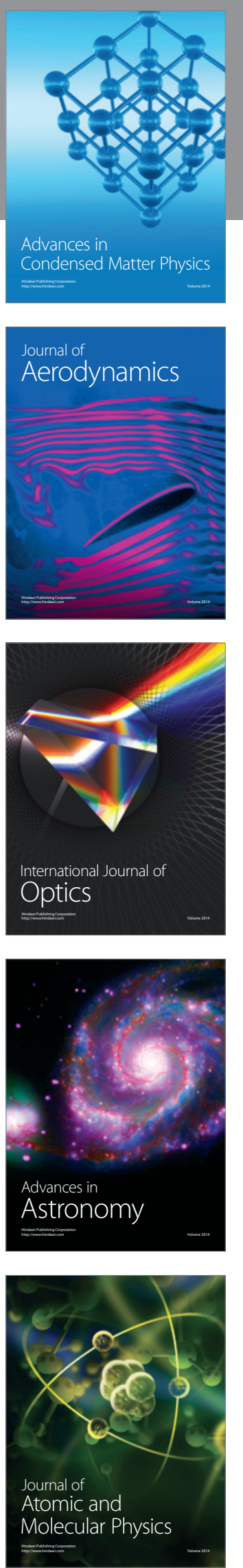